\title{
Liquid Fuel Evaporation under Supercritical
}

\section{Conditions}

\author{
Guowei Xiao ${ }^{1,2}$, Kai Hong Luo ${ }^{1,3^{*}}$, Xiao $\mathrm{Ma}^{2}$ and Shijin Shuai ${ }^{2}$ \\ ${ }^{1}$ Center for Combustion Energy, Tsinghua University, Beijing 100084, China
}

${ }^{2}$ State Key Laboratory of Automotive Safety and Energy, Tsinghua University, Beijing 100084, China

${ }^{3}$ Department of Mechanical Engineering, University College London, Torrington Place, London WC1E

7JE, UK

\begin{abstract}
Molecular dynamics simulations are performed to study the supercritical mixing process of the $n$ dodecane/nitrogen binary system. Previous studies have shown the existence of supercritical phenomenon under certain conditions in modern propulsion systems such as diesel engines. However, the physical mechanisms and internal driving forces of this phenomenon are still not well understood. In this paper, we attempt to answer this question through simulating the diffusion and evaporation of gaseous nitrogen and liquid phase $\mathrm{n}$-dodecane. It addresses under what conditions the supercritical transition phenomenon happens and what features the supercritical evaporation process have. A unique configuration is constructed to mimic the evaporation of an $\mathrm{n}$-dodecane thin film in an open nitrogen environment under conditions ranging from subcritical to supercritical. The detailed structure of the liquid-vapor interface during the evaporating process is described and the evaporation rate and the interface thickness are estimated, which show differences between subcritical and supercritical evaporation. Results indicate that under relatively high pressure conditions, the liquid surface transitions into supercritical state, and the liquid-vapor interface expands significantly with vanishing surface tension, leading to a diffusion like mixing process. It is shown that the supercritical evaporation would happen under conditions that correspond to the in-cylinder conditions of a turbo-charged engine.
\end{abstract}

\section{Introduction}

It has long been recognized that in some modern propulsion systems powered by spray combustion, such as diesel engines and liquid rocket combustion chambers, the environmental pressure can be higher than the critical pressure of the injected fuel. Considering the high temperature in the combustion chamber, it is reasonable to expect that the injected fuel may undergo a transition from liquid phase to supercritical phase, which can only diffuse but not evaporate. Therefore, our conventional knowledge on the atomization and combustion of the spray may be not sufficient to describe those processes under such conditions. In fact, a number of studies focused on this issue have found fundamentally different phenomena. Experimental studies [1-8] relevant to the conditions of liquid rocket combustion chamber showed that under high pressure and temperature conditions, the non-reacting sprays exhibit some characteristics similar to a turbulent gas jet, rather than the conventionally recognized breakup and evaporation phenomena. Chehroudi et al. [1] reviewed and plotted the experimentally measured and theoretically derived results regarding the initial spreading angle of both single-phase gas jet and 
two-phase sprays, as a function of the environment-to-injectant density ratio [9]. Quantitative agreement was confirmed between the measured results of two-phase sprays under near-critical conditions and the theoretical prediction for incompressible but variable-density gaseous jets by Dimotakis[10]. Besides that, smoothened liquid surface [11, 12] and the absence of ligaments or droplets [9, 13-17] were also observed and treated as signs of abnormal phenomena under supercritical conditions.

Despite these phenomenological descriptions and qualitative observations, our understanding of the supercritical injection process is still limited, due to the absence of effective quantitative measurement techniques. Most of the recent experimental studies have focused on the appearance and structural parameters of sprays, thus can provide little insight into the physical mechanism of these processes. In order to reveal the intrinsic features of this supercritical phenomena, especially the microscopic interfacial dynamics, more fundamental methods are desired.

Dahms et al. [18-21] made significant advances on this topic in their theoretical studies. A theoretical framework was presented to describe the thermodynamic structure of representative gas liquid interfaces under engine relevant conditions and predict the breakdown of classical twophase spray atomization theory. A real-fluid model together with vapor-liquid equilibrium theory and gradient theory was used to reconstruct the detailed structure of the vapor-liquid interface. A Knudsen number criterion was developed to quantify the conditions under which the interface region was in the continuum regime, and thus transition from classical two-phase interface to onephase mixing layer was initiated. In a recently published work [21], this framework was modified by applying the most modern form of the Helmholtz free energy equation of state and non-linear gradient theory and a generalized one-dimensional liquid injection model. The analysis showed that interfacial global thermal equilibrium is a prerequisite of vapor-liquid equilibrium relations and thus the classical two-phase theory. Thus it is concluded that the breakdown of the two-phase interface is initiated by the development of temperature gradient in the interfacial region, rather than the increased interfacial temperature. Consequently, a probability density function is derived to quantify under what conditions the temperature gradient can extend to the interfacial region and break the global thermal equilibrium. The $\mathrm{n}$-dodecane/nitrogen system was taken as an example and the presented regime diagram was in good agreement with corroborating experimental data by Crua et al. [22]. Works by Dahms et al. provide a theoretical framework for the conditions under which the injected diesel-like fuel shows a single phase diffusion-dominated mixing behavior, and to a certain extent explains the internal driving forces of this supercritical phenomenon.

Under such circumstances, there is interest in developing new models appropriate for spray combustion processes under supercritical conditions. Understanding the evaporation process of a relatively simple structure like an individual liquid droplet is considered to be essential for model development. A number of studies have investigated this issue using continuum-based numerical methods and experiments. Early in the 1990s, Curtis and Farrell [23] studied the evaporation of a single, spherical fuel droplet in nitrogen ambient numerically using a fully transient model, with real-gas effect and gas-phase diffusion into the droplet interior accounted for. The ambient pressure required for a droplet to reach the critical mixture state as a function of ambient temperature was obtained for a variety of fuels, and results showed good agreement with previous experiments of Sowls et al. [24]. The effect of transient vapor phase was investigated by comparing 
a simplified model assuming a quasi-steady vapor phase and the original model, the former one leads to a longer heat-up time and droplet lifetime of an $\mathrm{n}$-dodecane droplet. The same problem was further assessed by Zhu et al. [25]. In their work, the gas-phase unsteadiness was quantitatively defined as the ratio of the characteristic time for gas-phase transport and for surface dynamics, and was found to increase with increasing ambient pressure and/or temperature. Similar trend was confirmed by Aggarwal et al. [26]. The results of Zhu et al. [25] for n-heptane droplets evaporation into nitrogen environments showed that the quasi-steady gas phase assumption leads to a smaller evaporation rate initially and a larger evaporation rate during the later period, and significantly underestimated the minimum pressure needed for supercritical transition to happen. Stengele [27] considered multi-component droplets, the calculations for a single droplet consisting of $\mathrm{n}$-heptane and $\mathrm{n}$-dodecane evaporating in a high pressure and high temperature nitrogen ambient were carried out. The results with respect to the effects of pressure on the evaporation process were in consistent with single-component droplet studies (e.g., the numerical study of Zhu et al. [28] for n-heptane droplets): at a high ambient temperature the droplet lifetime decreases with higher pressures, and under low ambient temperature conditions, the lifetime first increases and then decreases with increasing pressures. Despite the considerable success in predicting the evaporation process, all of the above mentioned numerical studies, to a certain extent, adopted some property evaluation scheme that uses empirical formulas for low pressure conditions, which introduced inconsistencies into their model. Experimental investigations of the evaporation of a fuel droplet in high pressure and temperature ambient using microgravity conditions were used to validate the above mentioned models. The observations of Stengele et al. [27] and Zhu et al. [28] concerning the effects of ambient pressure on droplet lifetime were supported by Sato [29] for the evaporation of $n$-heptane droplet in nitrogen environment, but not consistent with the results of Nomura et al. for the same fuel [30] and nhexadecane [31], which stated that at low to moderate temperatures, droplet lifetime tends to increase monotonically with increasing pressure.

In the present work, molecular dynamics (MD) simulation is utilized to study the evaporation process of a liquid phase thin film of $n$-dodecane, the closest approximation to diesel fuel. MD is a direct simulation method for solving the equations of motion of all the interacting molecules in the system. Unlike the continuum-based methods, properties of the constituent substances in the system are calculated by statistics, hence no additional assumptions are necessary as long as an accurate intermolecular potential is provided. MD can be used regardless of phases or compositions, which makes it particularly suitable for the investigation of processes involving phase change and multi-component diffusion. However, MD simulations are computationally expensive, thus the system is always limited to small temporal and spatial scales, usually a few hundred nanoseconds and nanometers.

A number of MD studies have investigated the evaporation behavior of an individual liquid droplet [32-37] or a thin film [38,39], and various substances including argon and oxygen are considered. However, only a few of them reported observations under near- or super-critical conditions. Kaltz et al [34] studied the evaporation of a submicron liquid oxygen droplet into quiescent environments compromised of either gaseous hydrogen or helium. Significant effect of environmental pressure on the evaporation behavior was observed. At low to moderate pressures, the droplet remains spherical with a distinct surface during evaporation. While at extremely high pressures, the droplet evaporates in a cloud-like manner with vanishing surface tension. 
In this paper, we focus on this topic in the context of Diesel engine fuel injection processes. Recent experimental works $[18,40]$ showed that the transition from traditional spray atomization to a continuous turbulent mixing layer can also take place in diesel engine relevant conditions. By creating a liquid $\mathrm{n}$-dodecane thin film surrounded by nitrogen environment, the dynamic evaporation process of $n$-dodecane is studied. Besides the evaporation behavior, special efforts are paid to investigate the differences in interfacial phenomena of subcritical and supercritical conditions.

Since the solution of ambient gas into the injected liquid cannot be ignored at such high pressures, the determination of critical conditions of mixture is complex. For convenience, in what follows, the terms subcritical and supercritical refer to the critical conditions of the pure substance of $n$-dodecane and not the environment nor the mixture therein, unless otherwise stated.

\section{Model and Simulation}

A thin liquid film of $n$-dodecane and surrounding nitrogen molecules are considered in a three dimensional cuboid simulation box of size $150 \AA \times 150 \AA \times 2000 \AA$. Initially the liquid film consists of 20,763 n-dodecane molecules and is in contact with the nitrogen environment at the two interfaces. As illustrated in Fig. 1, periodic boundary conditions are used in all three directions, which allow nitrogen molecules that leave the box to reenter from the opposite side. It should be noted that periodic boundary conditions were only applied for nitrogen molecules. As will be discussed in detail below, $n$-dodecane molecules that move into the outmost regions in the $z$ direction are deleted. However, since boundary conditions in both $\mathrm{x}$ and $\mathrm{y}$ directions were periodic for both nitrogen and n-dodecane molecules, the present configuration would actually simulate the evaporation of an infinite-area fuel film into an open space.

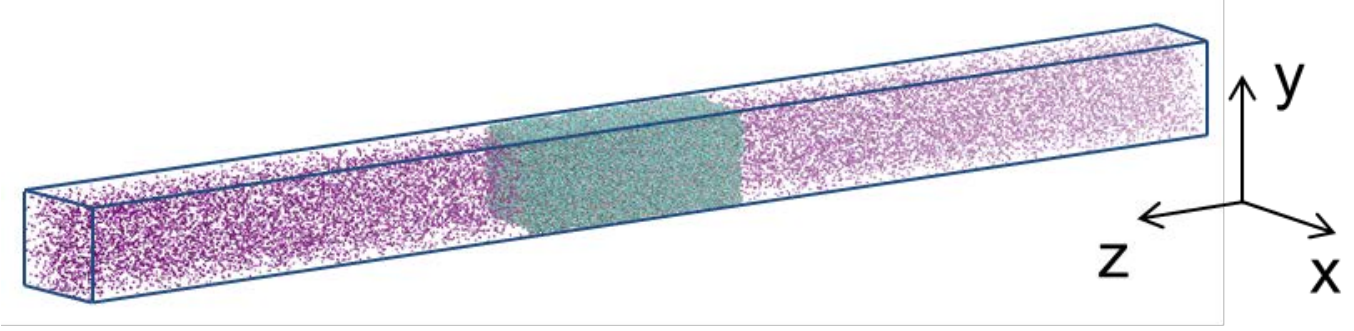

Fig. 1 Initial configuration of the n-dodecane/nitrogen binary system. The nitrogen molecules are in purple, and the $\mathrm{n}$-dodecane molecules are in green.

\subsection{Intermolecular potential}

A number of models have been developed to describe the intermolecular potentials of complex hydrocarbon molecules like $n$-dodecane [41-46] . Among them the so-called United Atom Models (UAM) are most widely utilized because of their balanced computational efficiency and accuracy. These models are based on the observation that the $\mathrm{C}-\mathrm{H}$ bond in complex hydrocarbon molecules is much shorter and stronger than the $\mathrm{C}-\mathrm{C}$ bond, thus methyl $\left(\mathrm{CH}_{3}\right)$ or methylene $\left(\mathrm{CH}_{2}\right)$ groups can be regarded as separate atom-like structures. Hydrogen atoms are not explicitly taken into account, hence an $\mathrm{n}$-dodecane molecule can be regarded as a chain containing 12 united atoms. For simplicity, we refer to united atoms as atoms in the following part.

Although the physical essence of various UAMs is the same, they differ by values of energy and size parameters for $\mathrm{CH}_{3}$ and $\mathrm{CH}_{2}$ groups. In this paper the applied model is a modified version based on that developed by Sanmartín et al. [46], which provides an accurate prediction of the thermodynamics properties of $n$-dodecane. The non-bonded interactions between atoms 
belonging to different chain or separated by three or more bonds if in the same chain are characterized by the truncated Lennard-Jones 12-6 potential, which has the form

$\mathrm{U}^{\mathrm{LJ}}\left(r_{i j}\right)=4 \varepsilon_{i j}\left\{\left[\left(\frac{\sigma_{i j}}{r_{i j}}\right)^{12}-\left(\frac{\sigma_{i j}}{r_{i j}}\right)^{6}\right]-\left[\left(\frac{\sigma_{i j}}{r_{c}}\right)^{12}-\left(\frac{\sigma_{i j}}{r_{c}}\right)^{6}\right]\right\}$

where the energy parameters are $\varepsilon_{\mathrm{CH}_{3}}=\varepsilon_{\mathrm{CH}_{2}}=0.112 \mathrm{kcal} / \mathrm{mol}$, the size parameters $\sigma_{\mathrm{CH}_{3}}=$ $\sigma_{\mathrm{CH}_{2}}=4.01 \AA, r_{i j}$ is the distance between atoms, and $r_{c}$ is the cut-off distance, which is set to $2.5 \sigma_{\mathrm{CH}_{3}}(\sim 10 \AA)$ in this study.

The interactions within an n-dodecane molecule consist of bond stretching, bond bending and bond torsion. Stretching takes place between two atoms directly bonded to each other. Here the harmonic potential is utilized

$\mathrm{U}^{\mathrm{s}}(r)=\mathrm{k}_{\mathrm{b}}\left(r-r_{0}\right)^{2}$

where $r$ is the distance between the two bonded atoms, $r_{0}=1.53 \AA$ is the equilibrium bond distance, and the stretching coefficient is $\mathrm{k}_{\mathrm{b}}=350 \mathrm{kcal} /\left(\mathrm{mol} \cdot \AA^{2}\right)$. Bond bending takes place between three neighboring atoms. Here the harmonic potential is used

$\mathrm{U}^{\mathrm{b}}(\theta)=\mathrm{k}_{\theta}\left(\theta-\theta_{0}\right)^{2}$

where the bending coefficient is $\mathrm{k}_{\theta}=60 \mathrm{kcal} /\left(\mathrm{mol} \cdot \mathrm{rad}^{2}\right)$, and equilibrium angle is $\theta_{0}=109^{\circ}$. Bond torsion takes place between four neighboring atoms, describing the vibration of the dihedral formed by these atoms. Here the OPLS style potential proposed by Watkins et al. [47] is used

$\mathrm{U}^{\mathrm{t}}(\phi)=\frac{1}{2} k_{1}(1+\cos \phi)+\frac{1}{2} k_{2}(1-\cos 2 \phi)+\frac{1}{2} k_{3}(1+\cos 3 \phi)+\frac{1}{2} k_{4}(1-\cos 4 \phi)$

where $k_{1}=1.411 \mathrm{kcal} / \mathrm{mol}, k_{2}=-0.271 \mathrm{kcal} / \mathrm{mol}, k_{3}=3.145 \mathrm{kcal} / \mathrm{mol}$, and $k_{4}=0$.

The model describing the intermolecular potential of nitrogen molecule is relatively simple when comparing with $n$-dodecane. The non-bonded interactions are again characterized by the truncated Lennard-Jones 12-6 potential shown in Equation 1, with parameters $\varepsilon_{N}=$ $0.07232 \mathrm{kcal} / \mathrm{mol}$ and $\sigma_{N}=3.32 \AA$. The distance between two bonded nitrogen atoms is constrained at $1.106 \AA$. The interactions between united atoms and nitrogen atoms are obtained according to the Lorentz-Berthelot mixing rule, $\varepsilon_{C H_{3}-N}=\sqrt{\varepsilon_{C H_{3}} \varepsilon_{N}}=0.0900 \mathrm{kcal} / \mathrm{mol}$, and $\sigma_{C_{3}-N}=\frac{\sigma_{N}+\sigma_{C H_{3}}}{2}=3.67 \AA$.

A vapor-liquid equilibrium calculation is carried out to quantify the validity of the presented intermolecular potentials using the configuration depicted by Fig. 1. In the equilibrium simulations, periodic boundary conditions are used in all three directions, Nose-Hoover thermostat (NVT ensemble) was performed to control the temperature of the system, the number of nitrogen molecules in the system is varied so that the equilibrium pressure can be varied. Simulation results regarding the equilibrium composition of the vapor and liquid phases under three temperatures are compared with the experimental data provided by García-Cordova et al. [49], as shown in Fig. 2. It can be observed that, the results show a trend of increasing nitrogen solubility in the liquid phase with higher pressure and temperature, which is in accordance with the experimental results. The predicted data fit well with the experimental data at lower temperatures and pressures, but they are less satisfactory at higher temperatures or pressures. Previous studies [36] have shown that by increasing the cut-off distance of the Lennard-Jones potential the volatility of the liquid phase can be reduced, which in our case can benefit the prediction of the model. Nevertheless, computational expense is significantly increased with increasing cut-off distance, hence, a value of $2.5 \sigma_{\mathrm{CH}_{3}}$ is used throughout the present study as a compromise. 


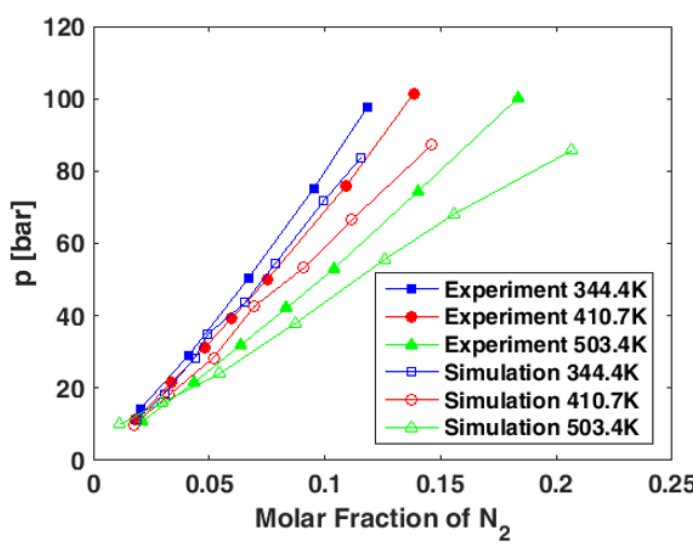

(a)

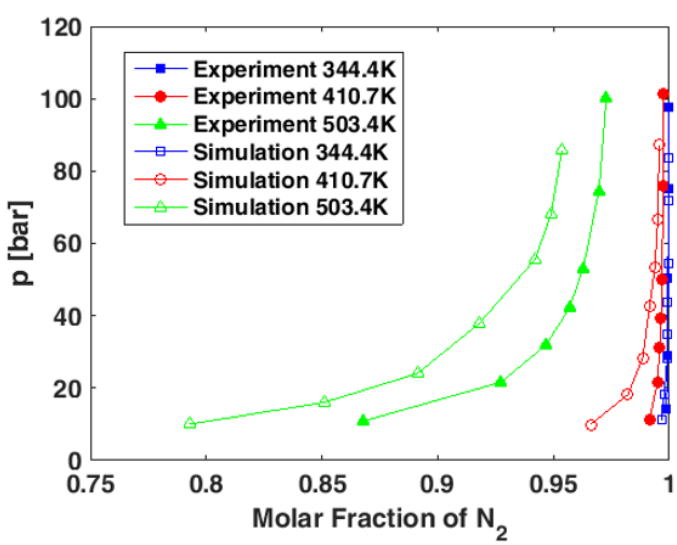

(b)

Figure 2: Pressure-composition phase diagram for the n-dodecane/nitrogen binary system: molar fractions of nitrogen in the (a) liquid phase and (b) vapor phase at vapor-liquid equilibrium conditions.

\subsection{System configuration}

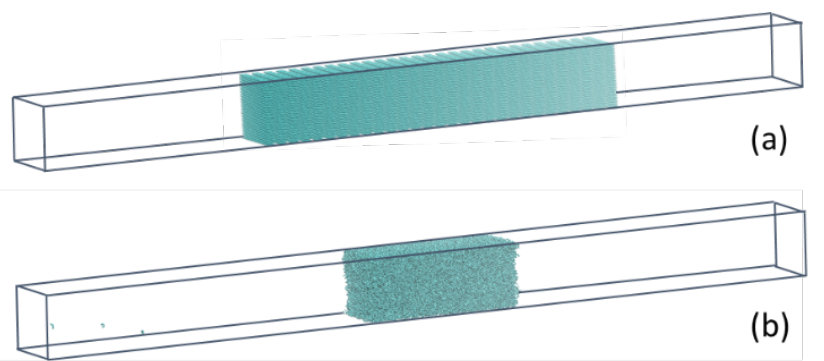

Figure 3: Snapshots of the equilibrium calculation of n-dodecane film: initial state (a) and equilibrium state (b)

A special model construction process was applied to prepare initial configuration of the dodecane-nitrogen binary system. Firstly, $20677(31 \times 23 \times 29$ in $x, y$ and $z$ dimension, respectively) $n$-dodecane molecules were placed in the middle of the simulation box and oriented along the $z$ axis (see Fig. 3a). Nose-Hoover thermostat (NVT ensemble) was performed to maintain a constant system temperature of 363K. The simulation ran for a sufficient time until the liquidvapor equilibrium condition was achieved (see Fig. 3b). A clustering method was used to identify liquid phase molecules. Those atoms which were at a distance shorter than a prescribed cutoff distance with each other were determined to be in the same 'cluster'. For every time steps, several clusters could be identified in the system, and the biggest one (which always contains much more molecules than others) must be the liquid film. Therefore, those molecules belonging to the biggest cluster was determined to be in liquid phase. The cutoff distance is believed to play a key role in this method, so several values were carefully compared and $7.0 \AA$ was shown to be adequate. At the equilibrium state, 20,673 n-dodecane molecules remained in liquid phase, and formed a liquid film of about $38 \mathrm{~nm}$ thick.

A gas phase nitrogen NVT simulation at the target temperature $T_{\text {tar }}$ was performed separately at the same time. Nitrogen molecules were initially placed on lattice points in the simulation box, and the total number is determined by the target environmental densities. As both the two simulations reached equilibrium, the saturated liquid-phase $n$-dodecane film was combined with the nitrogen environment, with the nitrogen molecules occupying the space of the 
liquid film deleted to avoid molecule overlap. See Fig. 4.

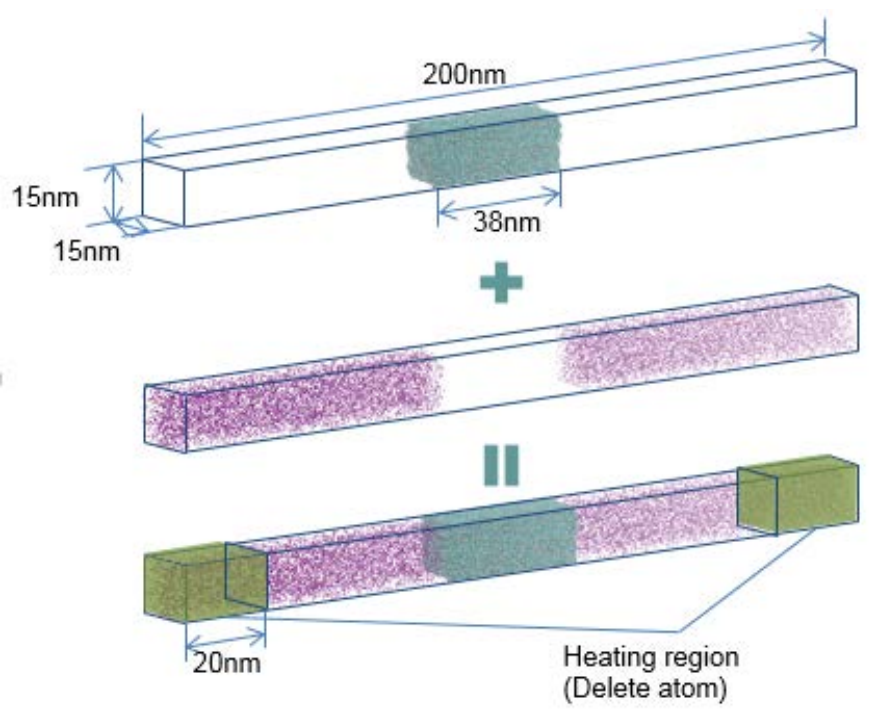

Figure 4: Snapshots of the construction process of the system initial configuration. The nitrogen molecules are in purple, and the $\mathrm{n}$-dodecane molecules are in green.

The simulations of the evaporation process were performed on the micro-canonical (NVE) ensemble. To mimic the evaporation of a liquid film in a constant environmental temperature, the 'far field' temperature was enforced at a target temperature by a simple velocity rescaling method: The two outermost side with a thickness of $20 \mathrm{~nm}$ in the $\mathrm{z}$ axis of the simulation box were defined as the 'heating region', in which the translational velocities of the molecules located were rescaled explicitly every timestep, according to

$v_{i}^{\text {new }}=v_{i}^{\text {old }} \sqrt{\frac{3 T_{\text {tar }} k_{B} N_{h}}{2 E_{\text {kin }}}}$

where $E_{\text {kin }}$ is the total kinetic energy of the $N_{h}$ atoms in the heating region, and $T_{\text {tar }}$ is the target environmental temperature. For all cases, the gaps between the film surface and the boundaries of heating regions were roughly $63 \mathrm{~nm}$, so surface dynamics were believed to be free from the boundary heating effects. To simulate evaporation, molecule deletion was applied in the heating region, that is to say, a molecule was removed from the simulation box once one of its atoms entered these regions. By this means, the liquid film was regarded to be evaporating in an open space, in which the evaporation process was not affected by the vapor phase $\mathrm{n}$-dodecane molecules.

The simulation details are summarized in Table 1. Temperature ranged from $600 \mathrm{~K}$ to $1100 \mathrm{~K}$, and pressure of each system was controlled by their respective initial environmental density, which was determined by the number of nitrogen molecules in the initial configuration. In NVE simulations, the pressure cannot be accurately controlled, thus it is difficult to make the pressures of different cases exactly equal. However, by carefully adjusting the number of nitrogen molecules in the system, we tried to make the ambient pressures as close as possible. The highest temperature (1100K) and pressure (99bar) were chosen since they were believed to be typical values of the in-cylinder condition of a turbo-charged diesel engine at the end of the compression stroke. The lowest temperature (600K) was chosen because it is below the critical value of $n$ dodecane, so definitively no supercritical transition would take place and this case can serve as a baseline case of the conventional low pressure evaporation behavior. It should be noted that 
because of the dissolution of nitrogen in the liquid phase and the cooling effect of evaporation, the pressure of the system suffered a noticeable decrease at the beginning of simulation, but during the following process, the pressure remained roughly constant. The actual pressures presented here are time-averaged values during the quasi-steady state period for all the cases.

The simulation was carried out with the Larger-scale Atomic/Molecular Massively Parallel Simulator (LAMMPS) code. The integration of the equations of motion is performed through the velocity Verlet algorithm with a time step of 2.0 femtoseconds for all the cases, and the total number of time steps varied for each simulation, depending on the evaporation time, the value ranged from 25,000,000 steps for Case 16 to 44,000,000 steps for Case 1 .

Table 1 Environmental conditions for the current simulations. The initial liquid phase n-dodecane was at saturated conditions at $363 \mathrm{~K}$ with a thickness of about $38 \mathrm{~nm}$.

\begin{tabular}{ccccc}
\hline $\begin{array}{c}\text { Case } \\
\text { Number }\end{array}$ & $\begin{array}{l}\text { Actual } \\
\text { Pressure [bar] }\end{array}$ & $\begin{array}{l}\text { Target } \\
\text { Temperature }[\mathrm{K}]\end{array}$ & $\begin{array}{l}\text { Number of } \\
\text { molecules }\end{array}$ & $\begin{array}{c}\text { n-dodecane } \\
\text { Total number of } \\
\text { molecules }\end{array}$ \\
\hline 1 & 35 & 600 & 20673 & 17003 \\
2 & 42 & 600 & 20673 & 19403 \\
3 & 66 & 600 & 20673 & 29465 \\
4 & 92 & 600 & 20673 & 39747 \\
5 & 41 & 750 & 20673 & 17052 \\
6 & 68 & 750 & 20673 & 25370 \\
7 & 92 & 750 & 20673 & 33626 \\
8 & 38 & 900 & 20673 & 15352 \\
9 & 43 & 900 & 20673 & 16975 \\
10 & 68 & 900 & 20673 & 22598 \\
11 & 92 & 900 & 20673 & 29223 \\
12 & 36 & 1100 & 20673 & 16105 \\
13 & 44 & 1100 & 20673 & 18068 \\
14 & 72 & 1100 & 20673 & 22787 \\
15 & 90 & 1100 & 20673 & 25011 \\
16 & 99 & 1100 & 20673 & 26169 \\
\hline
\end{tabular}

\section{Results and Discussion}

According to the above discussed configuration, the system is invariant in the $x$ and $y$ directions, hence the results presented here are averaged over the $x-y$ plane. Thermodynamics properties, e.g. temperature, density, etc. are calculated using statistical mechanics. In order to demonstrate the time-variation of those properties in the highly-dynamic evaporation process, data were presented every $4 \mathrm{~ns}(2,000,000$ steps). Each data point was the result of time-averaging over 40ps, which is adequate to provide good statistics as well as transient view of the system. 
The thermodynamic critical conditions of $\mathrm{n}$-dodecane and nitrogen are listed in Table 2 . Since the environmental pressures are higher than the critical value of $n$-dodecane (see Table 2), it is reasonable to expect that transition from liquid phase to supercritical phase can happen at the surface for the high temperature cases, which may lead to diffusion dominated mixing of $n$ dodecane with nitrogen and the disintegration of liquid-vapor interface.

Table 2 Critical conditions for $n$-dodecane and nitrogen

\begin{tabular}{ccc}
\hline & $\mathrm{T}_{\mathrm{Cr}}[\mathrm{K}]$ & $\mathrm{p}_{\mathrm{Cr}}[\mathrm{MPa}]$ \\
\hline N-dodecane & 658.1 & 1.817 \\
\hline Nitrogen & 126.2 & 3.396 \\
\hline
\end{tabular}

\subsection{Evaporation Behavior}

Usually the evaporation rate is presented as the evolution of the size of liquid phase, i.e., liquid film thickness. However, at the molecular level the film thickness is difficult to determine because of the existence of the interfacial region, which is a continuous change in density and composition during evaporation, especially under high pressure conditions. Hence we use the number of remaining molecules in liquid phase to indicate the evaporation rate.

Figure 5 shows the time-variation of the number of remaining molecules in liquid phase for the 16 cases. One general trend is that under higher environmental temperature, the liquid phase evaporates faster. However, the influence of environmental pressure on the evaporation rates seems to be uncertain. To better demonstrate that, the evaporation lifetime, which is defined as the time when $95 \%$ of the total amount of $n$-dodecane is evaporated, is plotted in Fig. 6 as a function of pressure for each ambient temperature condition. It is evident that, under low to moderate temperature conditions ( $600 \mathrm{~K}$ and $750 \mathrm{~K}$ ), the evaporation lifetime first decreases with increasing ambient pressure, reaches a minimum value, and then increases as pressure is further increased. Similar trend was observed in the numerical simulations of Yang et al. [49] and Mo et al. [50], in which liquid oxygen and n-dodecane were investigated, respectively. The decrease of evaporation lifetime under relatively low pressure is mainly caused by the decrease of the enthalpy of vaporization $L$, and as a result the decrease of mass transfer number $\left(B=c_{p}\left(T_{\infty}-T_{s}\right) / L\right)$ when ambient pressure is increased. Although the surface temperature $T_{S}$ is expected to increase, the variation is minor and cannot overtake the influence of decreasing $L$. When the pressure is further increased, as will be shown later, the evaporation becomes more diffusion-like; and increasing the ambient pressure causes the binary diffusion coefficient to decrease, which is even further decreased when more $n$-dodecane molecules are diffused into the ambient. As a result, the trend of pressure dependence is reversed. However, under higher temperature conditions (900K, 1100K), the evaporation lifetime decreases monotonically with pressure. As will be discussed later, supercritical transition happens during the evaporation process of the high pressure and high temperature cases. For the subcritical cases and the early stage of the transcritical cases, the influence of the decreasing enthalpy of vaporization still exists. When transition happens, the evaporation becomes completely diffusion-dominated, thus the effects of decreasing mass and heat diffusivity compensate the influence of decreasing enthalpy of 
vaporization, leading to a nearly pressure-independent evaporation lifetime.
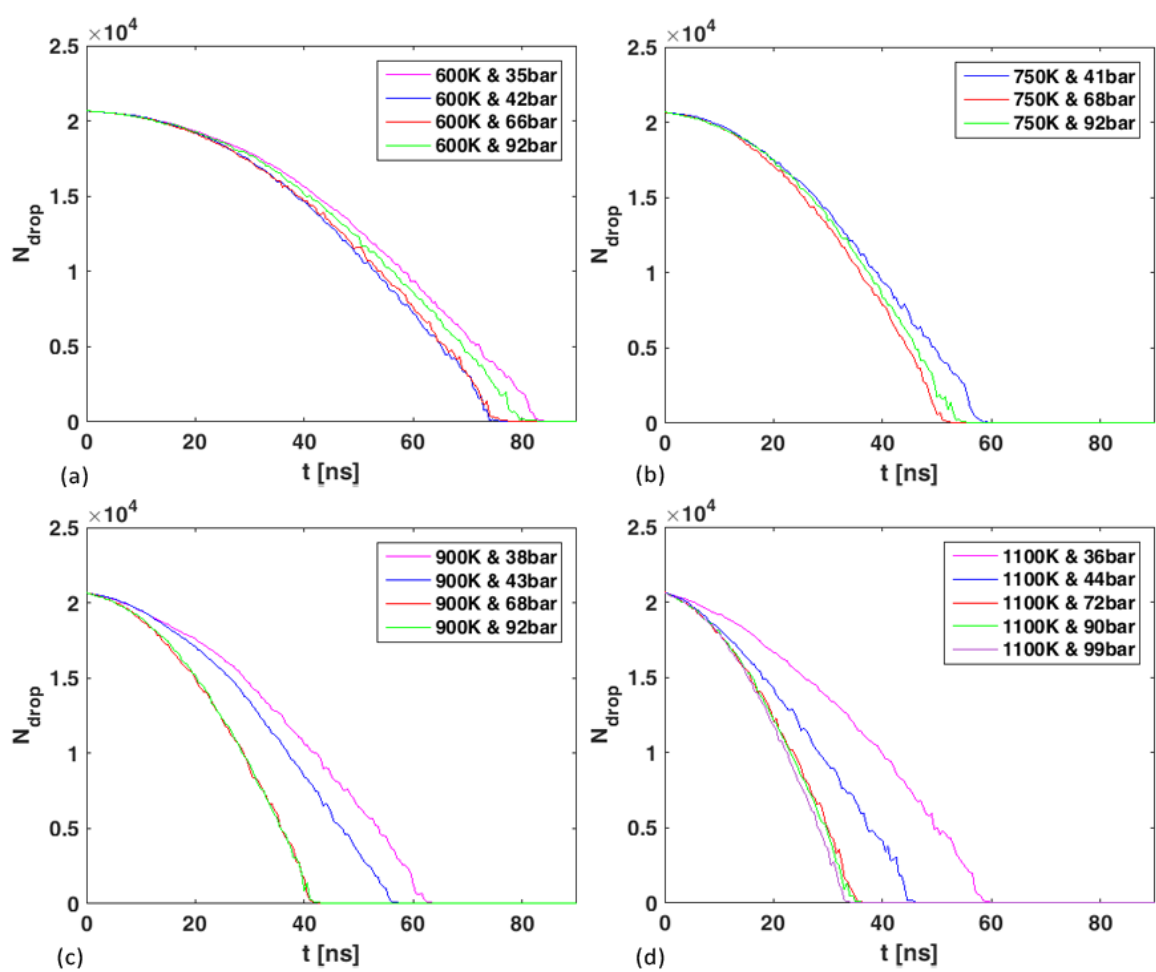

Figure 5: Time evolution of the number of remaining molecules in liquid phase at various environmental conditions.

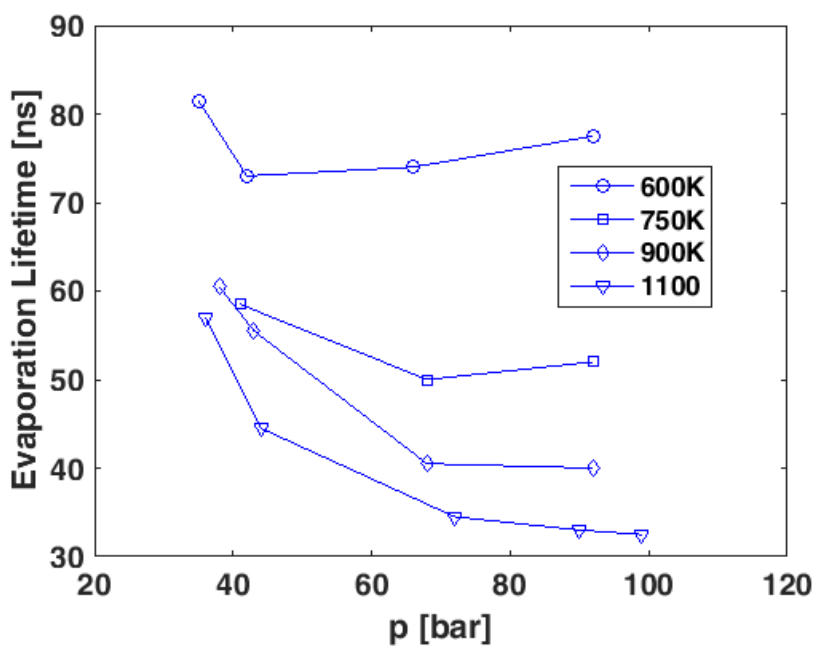

Figure 6: Evaporation lifetime of the liquid film as a function of ambient pressure

In order to determine the conditions under which supercritical transition happens, the temperature of the liquid surface is calculated and compared with the critical mixing temperature of $\mathrm{n}$-dodecane/nitrogen binary mixture, which is obtained from the experimental measurements of García-Cordova et al. [48]. Figure 7 shows the evolution of the surface temperature for the cases under low (600K) and high (1100K) temperature conditions. It is evident that for the two cases with high temperature and high pressure (case $141100 \mathrm{~K} \& 72 \mathrm{bar}$, case $151100 \mathrm{~K} \& 90 \mathrm{bar}$ ), the critical mixing temperature is reached before evaporation is completed. Thereupon, the distinct liquidvapor interface disappears, and the evaporation process afterwards is dominated by single-phase 
diffusion process, rather than the two-phase evaporation process in the conventional wisdom. A practical conclusion is that under conditions that correspond to the in-cylinder conditions of a turbo-charged engine, supercritical transition can happen, thus the validity of the existing spray models become questionable.
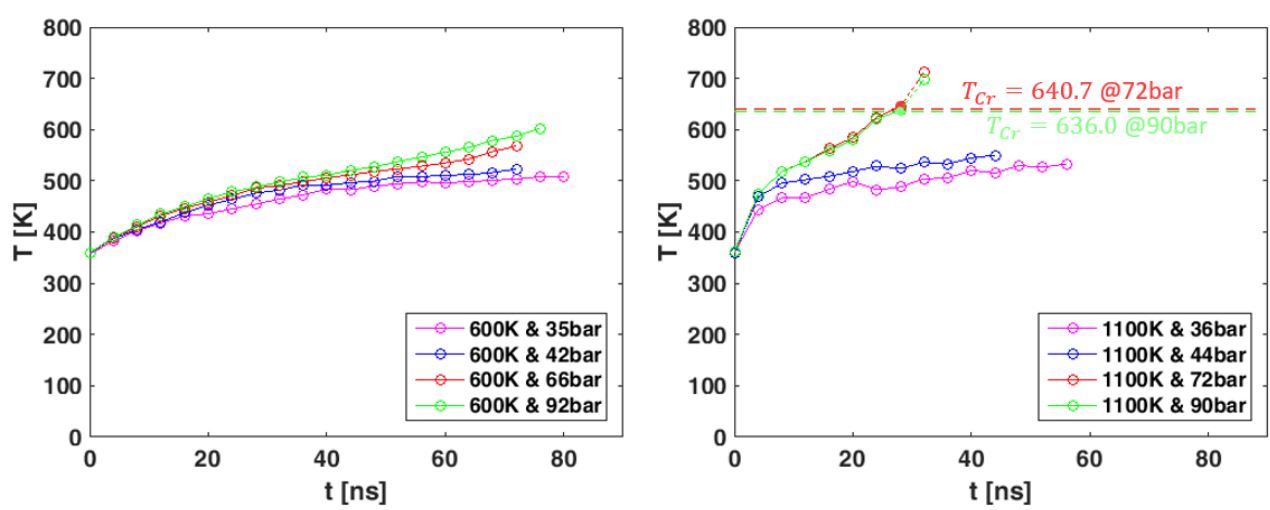

Figure 7: Time evolution of the surface temperature. Critical mixing temperature of the $\mathrm{n}$ dodecane/nitrogen binary system of the two supercritical cases are also shown in the figure.

As assumed in some conventional evaporation models under low pressure conditions, gas phase solution in the liquid phase is usually neglected [49]. However, under elevated pressures the solubility of nitrogen can be very large, which may lead to large deviation of model predictions from reality. MD simulations allow us to exam the validity of this assumption by calculating the number of nitrogen molecules penetrated into the liquid film. Figure 8 shows the variation of average nitrogen mole fractions in the central part of the liquid phase. For the lower pressure cases, the mole fraction of nitrogen maintains a roughly constant value of about 0.1 until the end of the lifetime of the liquid film. With increasing ambient pressure, a larger amount of nitrogen dissolves into the liquid phase. At the end of evaporation under high pressure comditions, as much as $80 \%$ of the liquid phase is made up of nitrogen molecules.
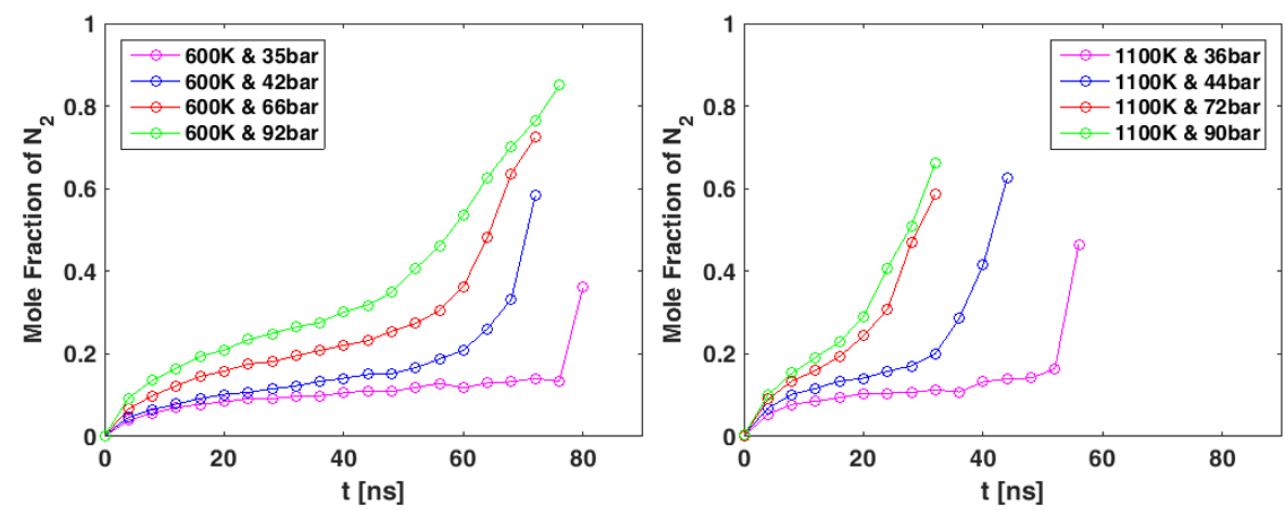

Figure 8: Time evolution of the nitrogen mole fraction in the liquid phase under environmental temperature of (a) 600K, (b) 1100K.

\subsection{Interfacial Properties}

Apart from the evaporation behaviors discussed above, previous studies have shown that 
differences between subcritical and supercritical evaporation also lie in the interfacial properties [34]. It is well documented that under low pressure conditions, evaporating liquid maintains a distinct density profile at the interface, while at very high pressures, as evaporation goes on, the liquid-vapor interface would expand, along with the disappearance of surface tension. In this section, we are going to discuss the evolution of the interface thickness and the surface tension. By comparing these two interfacial properties between different conditions, we can gain insight into the physical mechanism of the evaporation behavior under supercritical conditions.

Defining the edge of the liquid film on the molecular level is difficult due to the presence of the interfacial region between the liquid and gas, which is continuous in density and composition, as discussed in previous sections. Figure 9 shows the density profiles of $\mathrm{n}$-dodecane as a function of spatial distance during the evaporation processes for cases 1, 4, 12 and 15. Curves are plotted at $12 \mathrm{~ns}$ intervals. For case 1 and case 12 when the ambient pressure is low, the density profile at the interface region remains very sharp throughout the evaporation process, density of the liquid phase is much larger than the vapor phase, until a rapid decrease at the end of evaporation process (not shown in the figure). Comparing the results of higher pressure cases with the corresponding low pressure cases, a general trend is that the liquid phase expands significantly and the liquidvapor interface becomes smoother at the same time. Also the density of the vapor-phase $n$ dodecane in the vicinity of the interface is increased, which, as discussed in the previous section, reduced the binary mass diffusivity of the gas phase and the resulting evaporation rate.

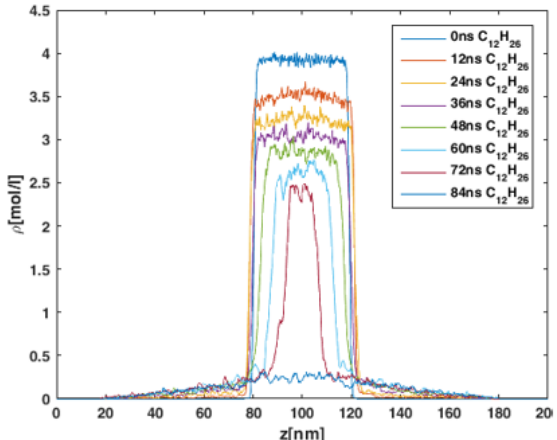

(a) $600 \mathrm{~K} \& 35 \mathrm{bar}$

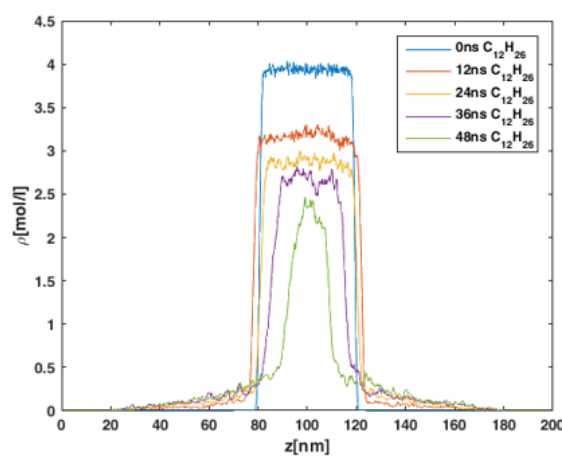

(c) $1100 \mathrm{~K} \& 36 \mathrm{bar}$

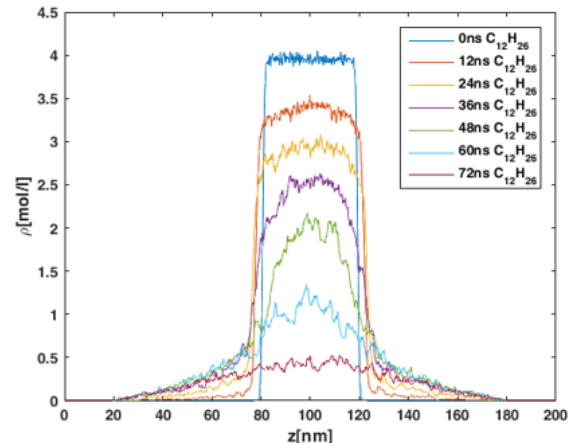

(b) $600 \mathrm{~K} \& 91 \mathrm{bar}$

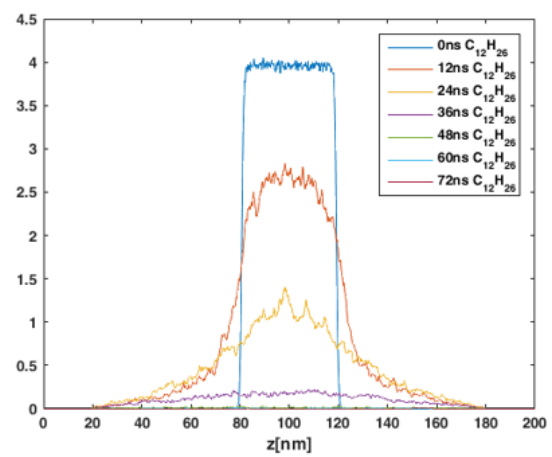

(d) $1100 \mathrm{~K} \& 90 \mathrm{bar}$

Figure 9: Time evolution of density profiles of $\mathrm{n}$-dodecane during the evaporation process under condition of (a) case1 600K \& 35bar, (b) case 4 600K \& 91bar, (c) case 12 1100K \& 36bar and (d) case 15 $1100 K$ \& 90bar.

By fitting the density curve to a hyperbolic tangent function, the thickness of the interface is 
determined by calculating the spatial distance of the $10^{\text {th }}$ percentile $\left(\rho=0.1 \rho_{l}+0.9 \rho_{g}\right)$ and $90^{\text {th }}$ percentile $\left(\rho=0.9 \rho_{l}+0.1 \rho_{g}\right)$ positions, as shown in Figure 10. The fitting function is given by [33]

$\rho(z)=\frac{\rho_{l}+\rho_{g}}{2}-\frac{\rho_{l}-\rho_{g}}{2} \tanh \left(\frac{z-z_{0}}{d}\right)$

where the fitting parameters $\rho_{l}, \rho_{g}$ and $z$ represent liquid phase density, gas phase density and the central position of the interface, respectively. The thickness of the interface equals to $2.2 d$, according to its definition. Figure 11 shows the thickness of the interfaces as a function of elapsed time for the cases under low (600K) and high (1100K) temperature conditions. At the beginning of the simulation, the thickness is about 1.6 nanometers under all conditions. Similar to the variation of nitrogen mole fraction in the liquid, for the low pressure cases, the thickness of the interface maintains a nearly constant value less than 10 nanometers, until a sharp increase at the end of evaporation, which may be ascribed to the rapid decrease of liquid density. While for the higher pressure cases, the thickness continually increases throughout the evaporation process.

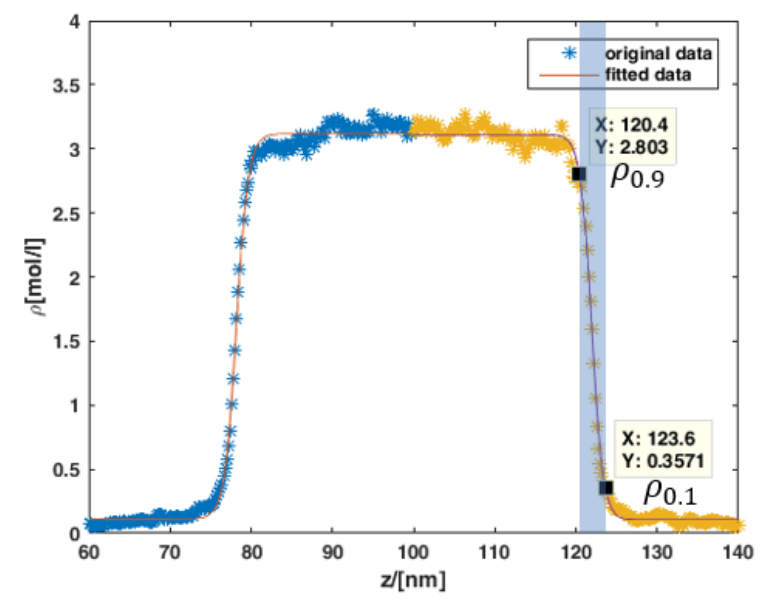

Figure 10: Illustration for the calculation of interface thickness, case $1600 \mathrm{~K} \& 35 \mathrm{bar}$ at $12 \mathrm{~ns}$

As shown in Figure 11, at the end of evaporation the interfaces for the moderate and high pressure cases are greatly expanded, thus it is natural to question whether the interfaces are still in the hydrodynamically discrete regime, or large enough to be treated as continuous phase. The Knudsen number of the interfaces are calculated. Here the Knudsen number is given by [19]

$\mathrm{Kn}=0.55 \frac{\lambda}{l}$

where $l$ is the thickness of interface, the coefficient 0.55 in the equation comes from the enhancement of molecular collisions due to the presence of an interface [21], $\lambda$ is the molecular mean free path of the gas phase in the vicinity of the surface, estimated by

$\lambda=\frac{1}{\sqrt{2} \pi\left(\frac{N}{V}\right) \sigma^{2}}$

where $\frac{N}{V}$ is the total number density and $\sigma$ is the average molecular size, which is given by

$\sigma=\sum_{i} X_{i} \sigma_{i}$

with $X_{i}$ as the species (n-dodecane and nitrogen in the present study) mole fraction and $\sigma_{i}$ as their Lennard-Jones size parameter. 

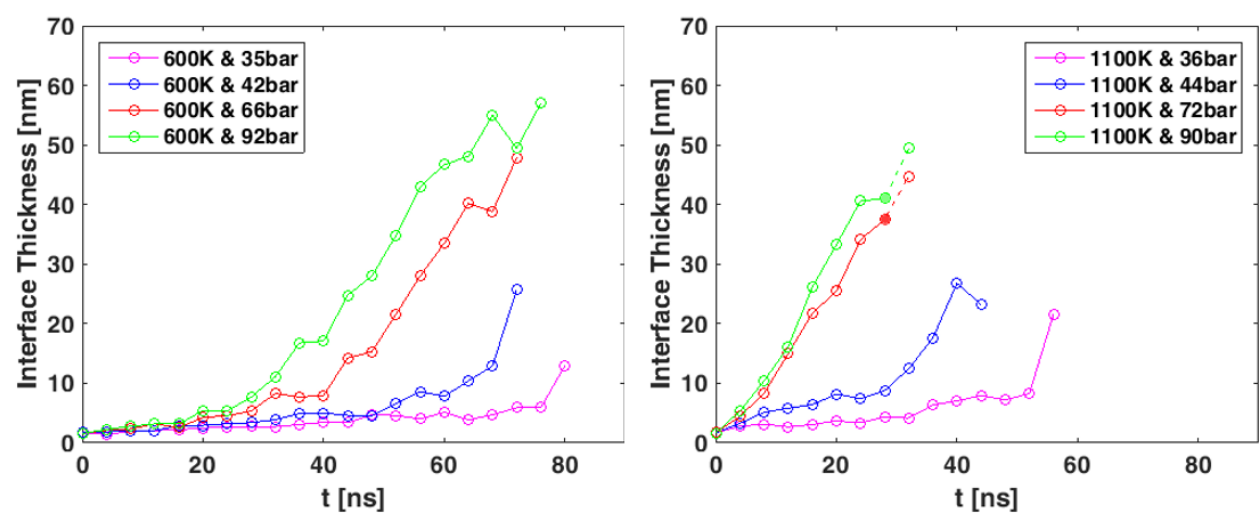

Figure 11: Time evolution of the interface thickness under environmental temperature of (a) 600K, (b) 1100K.

As shown in Figure 12, for the high pressure cases, the Knudsen number of the interface decreases to a value approaching 0.2. Though not sufficient to conclude that the interface is in the continuum regime, we can see that the Knudsen number has decreased greatly and the interface can no longer be treated as a discrete separation between the liquid and vapor phases. The critical mixing points, which are determined by calculating the surface temperature as discussed above, are also marked in Fig. 12. For the low temperature but high pressure conditions (e.g., case 4 600K \& 92bar), supercritical transition does not happen, even though the Knudsen number is as low as the cases with higher ambient temperatures. This observation agrees with the theoretical work of Dahms [21], in which it is claimed that the disintegration of the two-phase interface is initiated from the development of spatial interface temperature gradient. Under low temperature conditions (e.g. 600K), temperature difference between the liquid and vapor phase is small and thus spatial temperature gradient cannot develop. Therefore, the interface retains its thermal physical structure and surface tension still exists, as will be shown later. 


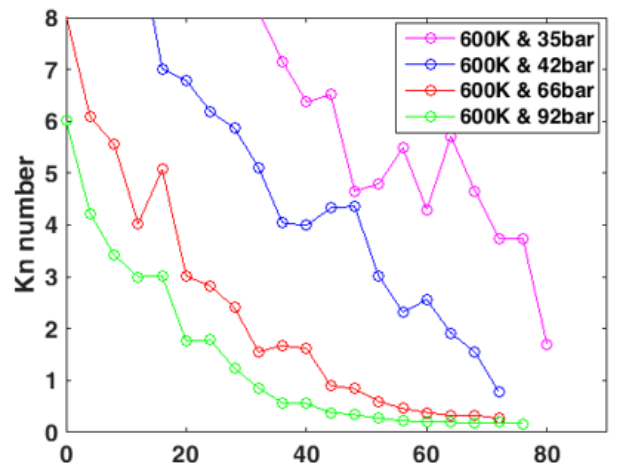

(a)

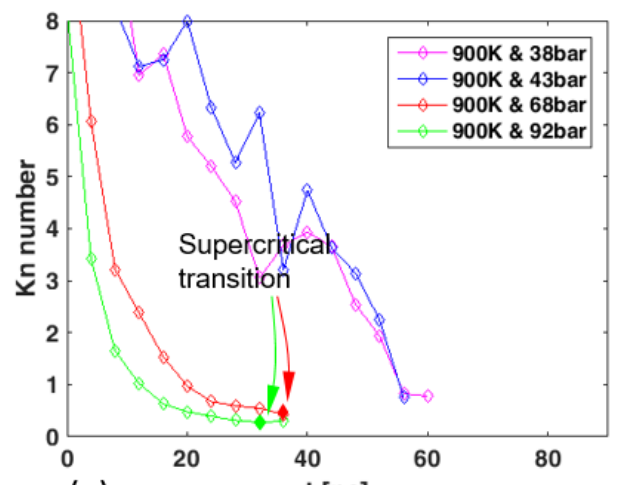

(c)

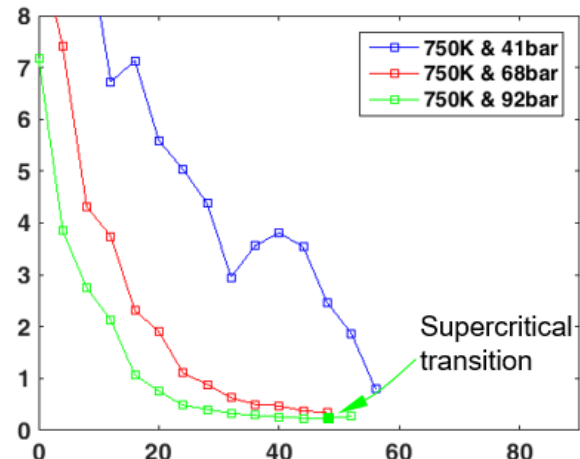

(b)

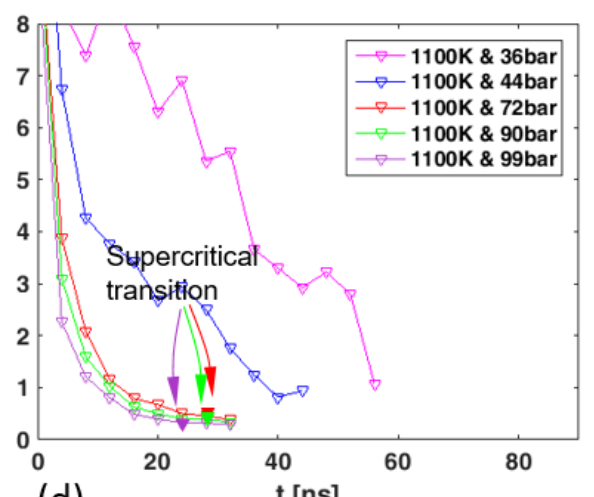

(d)

Figure 12: Time evolution of the Kn number of the liquid-vapor interface at various environmental conditions.

A number of molecular dynamics studies have reported the calculation of surface tension [38, 51-53], based on the observation that surface tension equals the spatial integral of the difference between the normal stress and the tangential stress at the interface. However, all of the previous studies were done at the equilibrium state. Actually it is intrinsically not feasible to calculate surface tension in the highly dynamic process of evaporation, because the calculation involves the rapidly-varying intermolecular forces. Usually the statistical errors induced by the fluctuating forces are reduced by time-averaging, which is not possible in the evolving systems.

An alternative method is to calculate the stress tensor of the fluid and estimate the difference between the normal and tangential components, which is an indication of surface tension and should be large in the region of a distinct interface. The stress tensor is calculated by

$S_{a b}=\frac{\sum_{N}\left[m v_{a} v_{b}+\frac{1}{2} \sum_{n=1}^{N p}\left(r_{1 a} F_{1 b}+r_{2 a} F_{2 b}\right)\right]}{V}$

where subscripts $a$ and $b$ denote the three spatial directions, $m$ is the mass of a particular atom, $r_{1 a}, r_{2 a}, F_{1 b}, F_{2 b}$ are the position vectors and pairwise forces of the atom and its neighbor, respectively. The equation includes a summation of the total energy of all the $N$ atoms in a region of volume $V$. The energies resulting from bond interactions, which fluctuate frequently and greatly in magnitude, can dominate over other forces, and thus are not included.

Figure 13 shows the difference between the normal and tangential stresses, $S_{x x}-\left(S_{y y}+\right.$ $\left.S_{z z}\right) / 2$, as a function of spatial position. Integrating the difference value along the $z$ axis gives the surface tension. However, due to the frequently vibrating intermolecular forces, the fluctuation in the liquid phase is very large, which make the accurate determination of surface tension not 
possible. Despite that, as indicated by the two peaks in the figure, the difference value in the interface region $(z \approx 80 n s \quad \& \quad z \approx 120 n s)$ is large at the beginning, when the interface is distinct. For case 1 and case 3 when the temperature is low, the difference in stresses is significant for a long time, which is an indication of strong surface tension throughout the duration of evaporation. However, for case 7, when the ambient temperature and pressure are both higher, the difference becomes negligible soon after the start of simulation. Uniformly distributed stress is acquired, which is a sign of vanished surface tension.

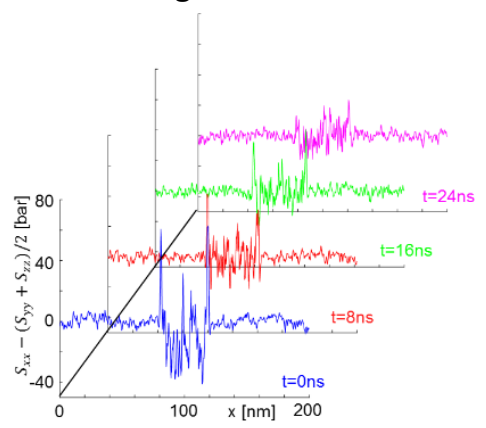

(a) Case $1,600 \mathrm{~K} \& 40 \mathrm{bar}$

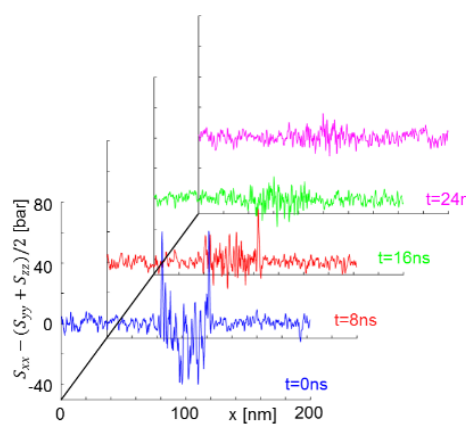

(b) Case 5 , 600K \& 66bar

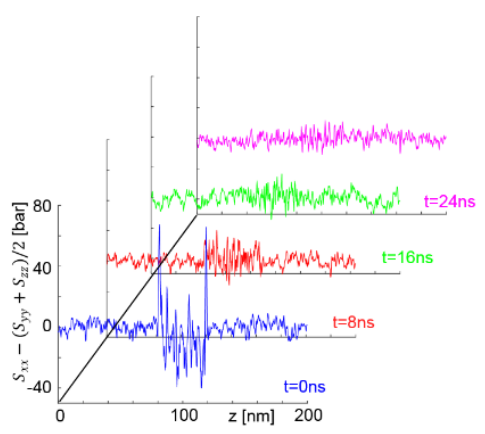

(c) Case 7, 900K \& 68bar

Figure 13: Time evolution of the difference between the normal and tangential stresses under condition of (a) 600K \& 35bar, (b) 600K \& 66bar, (c) 900K \& 68bar.

As discussed above, under relatively low pressure conditions, the existence of a distinct interface and strong surface tension indicate conventional subcritical evaporation behavior, although the pressure is indeed several times larger than the critical value of $n$-dodecane. Actually the critical pressure of binary mixtures, which depends on the composition, can be several times larger than each component. Recalling the dissolution of nitrogen into $n$-dodecane discussed in the previous section, it is easy to understand why supercritical transition did not happen in the low pressure cases. Under moderate and high pressure and high temperature conditions, evaporation proceeds in a fundamentally different manner, supercritical evaporation behavior is demonstrated by the increasing surface temperature and disappearing surface tension. Without the presence of a distinct liquid-vapor interface, traditional two-phase evaporation turns into a single-phase diffusion-like mixing.

\section{Conclusions}

The evaporation process of a long-chain alkane under supercritical conditions is studied by MD simulations. Supercritical evaporation behaviors of $n$-dodecane in nitrogen surrounding with respect to the evaporation rates and interfacial properties were observed and discussed.

Effects of environmental temperature and pressure on the evaporation behavior were investigated. It is shown that temperature greatly promotes evaporation due to the combination of enhanced mass and heat diffusion. However, the influence of pressure on the evaporation process is rather complex. Under low to moderate ambient temperature, the evaporation lifetime first decreases and reaches a minimum, then increases as pressure is further increased. While under higher temperatures, the evaporation lifetime decreases monotonically with pressure.

The conditions under which supercritical transition happens are quantified by determining whether the liquid surface reaches the critical mixing state during its lifetime. Under low pressure and low temperature conditions, transition from liquid phase to supercritical phase can never happen, the conventional evaporation behavior is observed as indicated by the existence of a welldefined liquid-vapor interface. While under moderate and high pressure and high temperature 
conditions, the liquid-vapor interface expands significantly with vanishing surface tension, leading to a diffusion like mixing process, rather than a conventional evaporation.

Beyond expectation, the conventional evaporation behavior with nearly constant liquid phase temperature and distinct liquid-vapor interface happens at pressures much higher than the critical pressure of the fuel, suggesting that quasi-steady approximation may be valid for a wide range of conditions.

\section{Acknowledgments}

Support from the Center for Combustion Energy at Tsinghua University are gratefully acknowledged. The simulations were performed on ARCHER funded under the EPSRC projects UK Consortium on Mesoscale Engineering Sciences (UKCOMES) (Grant No. EP/L00030X/1) and High Performance Computing Support for United Kingdom Consortium on Turbulent Reacting Flow (UKCTRF) (Grant No. EP/K024876/1).

\section{References}

[1]. Chehroudi, B., D. Talley, and E. Coy. "Visual characteristics and initial growth rates of round cryogenic jets at subcritical and supercritical pressures." Physics of Fluids 14, no. 2 (2002): 850-861.

[2]. Chehroudi, Bruce. "Recent experimental efforts on high-pressure supercritical injection for liquid rockets and their implications." International Journal of Aerospace Engineering 2012 (2012).

[3]. Tamura, H. "Injection and mixing processes in high-pressure liquid oxygen/gaseous hydrogen rocket combustors." Journal of Propulsion and Power 16, no. 5 (2000).

[4]. Oschwald, M., and A. Schik. "Supercritical nitrogen free jet investigated by spontaneous Raman scattering." Experiments in Fluids 27, no. 6 (1999): 497-506.

[5]. Oschwald, M. "Spreading angle and centerline variation of density of supercritical nitrogen jets." Atomization and Sprays 12, no. 1-3 (2002).

[6]. OSCHWALD*, M., J. J. Smith, R. Branam, J. Hussong, A. Schik, B. Chehroudi, and D. Talley. "Injection of fluids into supercritical environments." Combustion Science and Technology 178, no. 1-3 (2006): 49-100.

[7]. Mayer, Wolfgang OH, Axel HA Schik, Bruno Vielle, Christian Chauveau, Iskender Gökalp, Douglas G. Talley, and Rodger D. Woodward. "Atomization and breakup of cryogenic propellants under high-pressure subcritical and supercritical conditions." Journal of Propulsion and Power 14, no. 5 (1998): 835-842.

[8]. Mayer, W., J. Telaar, R. Branam, G. Schneider, and J. Hussong. "Raman measurements of cryogenic injection at supercritical pressure." Heat and Mass Transfer 39, no. 8 (2003): 709-719.

[9]. Chehroudi, B., D. Talley, and E. Coy. "Visual characteristics and initial growth rates of round cryogenic jets at subcritical and supercritical pressures." Physics of Fluids 14, no. 2 (2002): 850-861. [10]. DIMOTAKIS, PAULE. "Two-dimensional shear-layer entrainment." AIAA journal 24, no. 11 (1986): 1791-1796.

[11].Roy, Arnab, and Corin Segal. "Experimental study of fluid jet mixing at supercritical conditions." Journal of Propulsion and Power 26, no. 6 (2010): 1205.

[12]. Segal, C., and S. A. Polikhov. "Subcritical to supercritical mixing." Physics of Fluids 20, no. 5 (2008): 052101.

[13]. Oschwald, M., and A. Schik. "Supercritical nitrogen free jet investigated by spontaneous 
Raman scattering." Experiments in Fluids 27, no. 6 (1999): 497-506.

[14]. Oschwald, M. "Spreading angle and centerline variation of density of supercritical nitrogen jets." Atomization and Sprays 12, no. 1-3 (2002).

[15]. Branam, Richard, and Wolfgang Mayer. "Characterization of cryogenic injection at supercritical pressure." Journal of Propulsion and power 19, no. 3 (2003): 342-355.

[16]. Chehroudi, Bruce, and Douglas Talley. "The fractal geometry of round turbulent cryogenic nitrogen jets at subcritical and supercritical pressures." Atomization and Sprays 14, no. 1 (2004).

[17]. Woodward, Roger D., and Douglas G. Talley. "Raman imaging of transcritical cryogenic propellants." AIAA paper 468 (1996): 1996.

[18]. Dahms, Rainer N., Julien Manin, Lyle M. Pickett, and Joseph C. Oefelein. "Understanding highpressure gas-liquid interface phenomena in diesel engines." Proceedings of the Combustion Institute 34, no. 1 (2013): 1667-1675.

[19 Dahms, Rainer N., and Joseph C. Oefelein. "On the transition between two-phase and singlephase interface dynamics in multicomponent fluids at supercritical pressures." Physics of Fluids 25 , no. 9 (2013): 092103.

[20]. Dahms, Rainer N., and Joseph C. Oefelein. "Non-equilibrium gas-liquid interface dynamics in high-pressure liquid injection systems." Proceedings of the Combustion Institute 35, no. 2 (2015): 1587-1594.

[21]. Dahms, Rainer N. "Understanding the breakdown of classic two-phase theory and spray atomization at engine-relevant conditions." Physics of Fluids 28, no. 4 (2016): 042108.

[22]. Crua, C., J. Manin, and L. M. Pickett. "Transition from droplet evaporation to miscible mixing at diesel engine conditions." In 13th Triennial International Conference on Liquid Atomization and Spray Systems (ILASS-International, 2015). 2015.

[23]. Curtis, E. W., and P. V. Farrell. "A numerical study of high-pressure droplet vaporization." Combustion and Flame 90, no. 2 (1992): 85-102.

[24]. Sowls, R. E., Ph.D. thesis, Department of Mechanical Engineering, University of WisconsinMadison, 1972.

[25]. Zhu, Guang-Sheng, Rolf D. Reitz, and Suresh K. Aggarwal. "Gas-phase unsteadiness and its influence on droplet vaporization in sub-and super-critical environments." International Journal of Heat and Mass Transfer 44, no. 16 (2001): 3081-3093.

[26]. Aggarwal, Suresh K., Changlin Yan, and Guangsheng Zhu. "Transcritical vaporization of a liquid fuel droplet in a supercritical ambient." Combustion science and technology 174, no. 9 (2002): 103130.

[27]. Stengele, J., K. Prommersberger, M. Willmann, and S. Wittig. "Experimental and theoretical study of one-and two-component droplet vaporization in a high pressure environment." International journal of heat and mass transfer 42, no. 14 (1999): 2683-2694.

[28]. Zhu, G. S., and S. K. Aggarwal. "Transient supercritical droplet evaporation with emphasis on the effects of equation of state." International Journal of Heat and Mass Transfer 43, no. 7 (2000): 1157-1171.

[29]. Sato, J. "Studies on droplet evaporation and combustion in high pressures." (1993).

[30]. Nomura, Hiroshi, Yasushige Ujiie, Hans J. Rath, Jun'ich Sato, and Michikata Kono. "Experimental study on high-pressure droplet evaporation using microgravity conditions." In Symposium (International) on Combustion, vol. 26, no. 1, pp. 1267-1273. Elsevier, 1996.

[31]. Nomura, Hiroshi, Takahiro Murakoshi, Yusuke Suganuma, Yasushige Ujiie, Nozomu Hashimoto, 
and Hiroyuki Nishida. "Microgravity experiments of fuel droplet evaporation in sub-and supercritical environments." Proceedings of the Combustion Institute 36, no. 2 (2017): 2425-2432. [32]. Consolini, Lorenzo, Suresh K. Aggarwal, and Sohail Murad. "A molecular dynamics simulation of droplet evaporation." International journal of heat and mass transfer 46, no. 17 (2003): 31793188.

[33]. Long, Lyle N., Michael M. Micci, and Brian C. Wong. "Molecular dynamics simulations of droplet evaporation." Computer Physics Communications 96, no. 2-3 (1996): 167-172.

[34]. Kaltz, T. L., L. N. Long, M. M. Micci, and J. K. Little. "Supercritical vaporization of liquid oxygen droplets using molecular dynamics." Combustion science and technology 136, no. 1-6 (1998): 279301.

[35]. Walther, Jens Honore, and P. Koumoutsakos. "Molecular dynamics simulation of nanodroplet evaporation." Journal of Heat Transfer 123, no. 4 (2001): 741-748.

[36].Sumardiono, Siswo, and Johann Fischer. "Molecular simulations of droplet evaporation processes: Adiabatic pressure jump evaporation." International journal of heat and mass transfer 49, no. 5 (2006): 1148-1161.

[37]. R. Nasiri and K. H. Luo, "Specificity switching pathways in thermal and mass evaporation of multicomponent hydrocarbon droplets: a mesoscopic observation," Scientific Reports (2017, In press). DOI: 10.1038/s41598-017-05160-z

[38]. Cheng, Shengfeng, Jeremy B. Lechman, Steven J. Plimpton, and Gary S. Grest. "Evaporation of Lennard-Jones fluids." The Journal of chemical physics 134, no. 22 (2011): 224704.

[39]. Yi, Pan, D. Poulikakos, J. Walther, and G. Yadigaroglu. "Molecular dynamics simulation of vaporization of an ultra-thin liquid argon layer on a surface." International Journal of Heat and Mass Transfer 45, no. 10 (2002): 2087-2100.

[40]. Falgout, Zachary, Mattias Rahm, Zhenkan Wang, and Mark Linne. "Evidence for supercritical mixing layers in the ECN Spray A." Proceedings of the Combustion Institute 35, no. 2 (2015): 15791586.

[41]. Paul, Wolfgang, Do Y. Yoon, and Grant D. Smith. "An optimized united atom model for simulations of polymethylene melts." The Journal of chemical physics 103, no. 4 (1995): 1702-1709. [42]. Smit, Berend, Sami Karaborni, and J. Ilja Siepmann. "Computer simulations of vapor - liquid phase equilibria of $n$ - alkanes." The Journal of chemical physics 102, no. 5 (1995): 2126-2140.

[43].Xie, Jian-Fei, Sergei S. Sazhin, and Bing-Yang Cao. "Molecular dynamics study of the processes in the vicinity of the n-dodecane vapour/liquid interface." Physics of Fluids 23, no. 11 (2011): 112104.

[44]. Cao, Bing-Yang, Jian-Fei Xie, and Sergei S. Sazhin. "Molecular dynamics study on evaporation and condensation of $n$-dodecane at liquid-vapor phase equilibria." The Journal of chemical physics 134, no. 16 (2011): 164309.

[45]. Jorgensen, William L., Jeffry D. Madura, and Carol J. Swenson. "Optimized intermolecular potential functions for liquid hydrocarbons." Journal of the American Chemical Society 106, no. 22 (1984): 6638-6646.

[46]. Sanmartín, Sara, Javier Ramos, Juan Francisco Vega, and Javier Martínez-Salazar. "Strong influence of branching on the early stage of nucleation and crystal formation of fast cooled ultralong n-alkanes as revealed by computer simulation." European Polymer Journal 50 (2014): 190-199.

[47]. Watkins, Edward K., and William L. Jorgensen. "Perfluoroalkanes: Conformational analysis and 
liquid-state properties from ab initio and Monte Carlo calculations." The Journal of Physical Chemistry A 105, no. 16 (2001): 4118-4125.

[48]. García-Córdova, Tomás, Daimler N. Justo-García, Blanca E. García-Flores, and Fernando García-Sánchez. "Vapor- Liquid Equilibrium Data for the Nitrogen+ Dodecane System at Temperatures from (344 to 593) $\mathrm{K}$ and at Pressures up to $60 \mathrm{MPa}$." Journal of Chemical \& Engineering Data 56, no. 4 (2011): 1555-1564.

[49]. Yang, Vigor, and JIAN-SHUN SHUEN. "Vaporization of liquid oxygen (LOX) droplets in supercritical hydrogen environments." Combustion Science and Technology 97, no. 4-6 (1994): 247-270.

[50]. Mo, Guiyuan, and Li Qiao. "A molecular dynamics investigation of n-alkanes vaporizing into nitrogen: transition from subcritical to supercritical." Combustion and Flame 176 (2017): 60-71.

[51]. Shi, Bo, Shashank Sinha, and Vijay K. Dhir. "Molecular dynamics simulation of the density and surface tension of water by particle-particle particle-mesh method." The Journal of chemical physics 124, no. 20 (2006): 204715.

[52]. Langroudi, SM Mirnouri, M. Ghassemi, A. Shahabi, and H. Rezaei Nejad. "A molecular dynamics study of effective parameters on nano-droplet surface tension." Journal of Molecular Liquids 161, no. 2 (2011): 85-90.

[53] Rezaei Nejad, H., M. Ghassemi, S. M. Mirnouri Langroudi, and A. Shahabi. "A molecular dynamics study of nano-bubble surface tension." Molecular Simulation 37, no. 01 (2011): 23-30. 